\title{
Menstrual Disorder
}

National Cancer Institute

\section{Source}

National Cancer Institute. Menstrual Disorder. NCI Thesaurus. Code C34815.

A category of conditions related to menses. 